\title{
UN MODELO ECOLÓGICO INTEGRADO PARA COMPRENDER LA VIOLENCIA CONTRA LAS MUJERES
}

\author{
CARMEN Vives CASES \\ Universidad de Alicante ${ }^{1}$
}

\begin{abstract}
Recibido/15/07/2011
\end{abstract}
Aceptado/25/09/2011

\section{Referencia del artículo comentado}

HEISE, Lory L. «Violence against women: An integrated, ecological framework». Violence against Women 1998; 4: 262-290.

\section{Resumen}

Este artículo fomenta la adopción general de un marco integrado ecológico para la comprensión de los orígenes de la violencia de género. La aproximación ecológica al maltrato conceptualiza la violencia como un fenómeno polifacético basado en la interacción entre factores personales, situacionales y socioculturales. A pesar de tratar los avances conceptuales de teóricos anteriores, este artículo va más allá de su trabajo en tres aspectos relevantes. En primer lugar, este artículo utiliza el marco ecológico como una herramienta heurística para organizar la base de investigación existente como un todo inteligible. Mientras que otros teóricos presentan el marco como una manera de pensar sobre la violencia, unos pocos han intentado establecer qué factores surgen como predictores del maltrato en cada uno de los niveles de la ecología social. En segundo lugar, este artículo integra resultados de investigación internacionales y transculturales junto con descubrimientos de la ciencia social de Norteamérica. Y finalmente, el marco parte de descubrimientos relativos a todo tipo de maltrato físico y sexual hacia las mujeres para fomentar una aproximación más integrada a la construcción de teorías en cuanto al maltrato hacia las mujeres.

1. Pertenece al Grupo de Investigación en Salud Pública de la Universidad de Alicante.

Feminismo/s 18, diciembre 2011, pp. 291-299 


\section{Introducción}

La violencia de género contra las mujeres es una vulneración de los derechos humanos que obstaculiza el logro de la igualdad, la paz y el desarrollo². También es un grave problema de Salud Pública por sus devastadoras consecuencias para la salud de las mujeres incluida la mortalidad por esta causa. En este sentido, se ha puesto de manifiesto que su eliminación podría ser una importante contribución al desarrollo a nivel mundial, que actualmente se concreta en los denominados «Objetivos del Milenio» de Naciones Unidas que abarcan desde promover la igualdad y la autonomía de las mujeres hasta la reducción de la mortalidad infantil, materna y de la propagación de VIH/SIDA. 3,4

La violencia de género también es una de las consecuencias de la desigualdad de género relacionada con los valores, normas y creencias sobre la posición de las mujeres en la sociedad. El Patriarcado, que es identificado como el sistema de valores, normas y creencias que retroalimenta esta desigualdad de género en la cultura y la sociedad, tiene diferentes formas y grados ${ }^{5}$. Puede decirse, por tanto, que la desigualdad de género impregna todos los niveles de determinantes de la salud de la población que abarcan desde el individual al del contexto social.

En la última década, parece de común acuerdo la necesidad de explicar la violencia de género en función de las características del contexto social, del entorno más próximo a los hechos violentos y de las características de las personas afectadas. En este sentido, Lory L. Heise propuso en 1998 un marco ecológico integrado para la explicación de la violencia contra las mujeres en el que se conceptualiza la violencia como un fenómeno polifacético que se asienta en la interacción entre los factores personales, situacionales y

2. KRANTZ, Gunilla; GARCIA-MorenO, Claudia. «Violence against women». Journal of Epidemiology and Community Health 59 (2005), pp. 818-821.

3. ELLSBERG, Mary. «Violence against women and the Millennium Development Goals: Facilitating Women's access to support». Internacional Journal of Gynecology and Obstretics 94 (2006), pp. 325-332.

4. <http://www.un.org/spanish/millenniumgoals/>, consultado el 18-04-2011.

5. WALBY, Sylbia. Theorising Patriarchy. Oxford, Blackwell, 1990.

Feminismo/s 18, diciembre 2011, pp. 291-299 
socioculturales que pueden actuar como determinantes en este problema ${ }^{6}$. Se trata de uno de los modelos teórico-explicativos de la violencia contra las mujeres más ampliamente reconocido en la investigación sobre este tema desarrollada en el ámbito de la ciencias de la salud.

\section{Desarrollo}

En el artículo de Lory L. Heise, al que hacemos referencia, se propone un marco ecológico explicativo de la violencia contra las mujeres con el fin de integrar los hallazgos de diferentes displinas que han teorizado sobre las posibles causas de este problema. Se basa en una revisión de la investigación académica de Estados Unidos y Canadá desde las perspectivas de la antropología, la psicología y la sociología. Como resultado, la autora propone un modelo de determinantes de la violencia contra las mujeres distribuido en cuatro niveles de la ecología social:

1. Nivel 1: Historia Personal

- Presenciar violencia conyugal en la infancia

- Sufrir malos tratos durante la infancia

- Padre ausente o que rechaza a su hijo/a

2. Nivel 2: Microsistema

- Dominación masculina en el seno familiar

- Control masculino del patrimonio familiar

- Consumo de alcohol

- Conflicto conyugal/ verbal

3. Nivel 3: Exosistema

- Bajo status socioeconómico/ desempleo

- Aislamiento de la mujer y la familia

- Grupos delictivos de iguales

4. Nivel 4: Macrosistema

- Derecho/ propiedad del hombre sobre la mujer

- Masculinidad asociada a la dominación y agresión

- Roles de género rígidos

- Aprobación de la violencia interpersonal

- Aprobación del castigo físico

6. HEISE, Lory L. «Violence against women: An integrated, ecological framework» Violence against Women 4 (1998), pp. 262-290. 
Tal y como pone de manifiesto la autora del artículo, este marco teórico no debe ser interpretado como definitivo, puesto que, se basa en un conjunto provisional e incompleto de factores que han sido relacionados previamente en la literatura científica con la violencia contra las mujeres. En este sentido, se propone orientar el análisis del artículo propuesto en la explicación de algunos de los factores identificados en el modelo, así como de la calidad de la literatura científica que se ha generado en torno a los mismos.

\subsection{Alcohol y violencia de género. Una relacion sobrevalorada}

Muchos investigadores creen que el alcohol juega un importante papel en la desinhibición del agresor en ciertos tipos de abusos, ofuscando su juicio y afectando a su capacidad de interpretar las respuestas de la otra persona. No obstante, aún se desconoce cómo actúa el alcohol para incrementar el riesgo de que un hombre desarrolle específicamente una conducta violenta contra su pareja. De hecho, un buen número de hombres alcohólicos no maltratan físicamente a sus parejas y no todos aquellos que lo hacen, abusan del alcohol.

En un artículo de revisión de estudios publicados sobre la relación entre consumo de alcohol excesivo y violencia contra las mujeres, se pone de manifiesto que los estudios desarrollados en torno a este tema presentan algunas limitaciones relacionadas con la falta de precisión de las asociaciones encontradas y la tendencia de las revistas científicas a publicar los resultados positivos más que los negativos ${ }^{\top}$.

Resulta todavía más preocupante que el consumo excesivo de alcohol pueda proporcionar una excusa que permita a los agresores eludir su responsabilidad por su conducta agresiva. Esta realidad ha llevado a muchas activistas feministas y a quienes toman decisiones políticas a mostrar cautela a la hora de reconocer el papel del alcohol en la etiología de este tipo de abuso.

\subsection{El aprendizaje intergeneracional de la violencia y de la indefensión ante los abusos}

Con respecto a la historia personal de los individuos, el aprendizaje intergeneracional de la violencia contra las mujeres es uno de los factores que mayor preocupación despierta en todas sus formas: por haber recibido malos tratos en la infancia, por haber atestiguado en la infancia la violencia en la pareja o por rechazo de la figura paterna. Es reconocido como uno de los factores

7. Gil-GonZÁlez, Diana; Vives-Cases, Carmen; Álvarez-Dardet, Carlos; Latour-PéreZ, Jaime. «Alcohol and intímate partner violence: do we have enough information to act?» European Journal of Public Health 16 (2006): 278-284.

Feminismo/s 18, diciembre 2011, pp. 291-299 
de riesgo más consistentes en relación con la conducta violenta de hombres contra mujeres ${ }^{8}$. Sin embargo, una revisión sistemática reciente pone de manifiesto la necesidad de mejorar la calidad de las investigaciones en esta línea, puesto que, todavía son escasos los estudios con diseños adecuados -por ejemplo, estudios de cohortes o casos y controles- para analizar esta relación. Las investigaciones existentes también se ven limitadas por la presencia de sesgos epidemiológicos importantes, como el de recuerdo, y por la heterogeneidad de escalas utilizadas para medir la exposición a este factor ${ }^{9}$. Una de las escalas más utilizadas para medir malos tratos en la infancia como factor de riesgo para la conducta violenta en hombres adultos es la denominada «Conflict Tactic Scale» (CTS). En sus primeras versiones, se incluía conjuntamente atestiguar y sufrir malos tratos en la infancia. Se trata de una medición metodológicamente incorrecta, que no permite distinguir entre la presencia de violencia en la pareja o análogo en la infancia (que es violencia sexista) y haber sufrido malos tratos durante la infancia (que es violencia patriarcal) ${ }^{10}$.

Una de las inquietudes más comunes es la incapacidad para responder por qué el aprendizaje intergeneracional de la violencia se manifiesta de forma diferente entre hombres y mujeres. Así, las niñas víctimas de violencia parecen mostrar una mayor probabilidad de ser de nuevo víctimas al llegar a la etapa adulta y los hombres tienen mayor riesgo de ser maltratadores. Esta tendencia podría explicarse por el impacto de la desigualdad de género en la socialización de los individuos y la asunción de roles diferentes por parte de los hombres y las mujeres ${ }^{11}$.

\subsection{Violencia contra las mujeres, conflicto verbal y dominación masculina}

Con respecto a los factores de riesgo ligados al contexto inmediato en el que se producen los hechos violentos, destacan el conflicto verbal en la pareja y

8. Hotaling, GT; SUGARMAN DB. «An analysis of risk markers in husband to wife violence: the current state of knowledge». Violence and Victims 1 (1986), pp. 101-124.

9. Gil-GonzÁlez, Diana; Vives-Cases, Carmen; Ruiz-CAnTERo, Maria Teresa; CarrascoPortiÑo, Mercedes; ÁlvareZ-DARDET, Carlos. «Childhood experiences of violence in prepetrators as a risk factor of intimate partner violence: a systematic review». Journal of Public Health 30 (2007), pp. 14-22.

10. Ruiz-Cantero, Maria Teresa; Vives-Cases, Carmen; Artazcoz, Lucía; Delgado, Ana; García-Calvente, Maria del Mar; Miqueo, Carmen; Montero, Isabel; Ortiz, Rocío; RONDA, Elena; RuIZ, Isabel; VALLS, Carmen. «A framework to analyse gender bias in epidemiological research». Journal of Epidemiologic and Community Health 61 (Suppl 2), pp. 46-53.

11. JASINSKI, Jane: «Theoretical Explanations for Violence Against Women», en Renzetti, C; Edleson, J; Berger R (dirs.): Sourcebook on Violence against women, California, Sage Publications, 2001, pp. 5-21. 
la dominación masculina sobre los aspectos que afectan al núcleo familiar por su relación con la violencia de género contra las mujeres en la pareja ${ }^{6}$. El efecto de ambos factores sobre la violencia contra las mujeres parece producirse fundamentalmente cuando los hombres muestran actitudes proclives a la ideología patriarcal que sustenta la subordinación de las mujeres hacia los hombres ${ }^{12}$.

Aportaciones más recientes introducen en esta relación conflictiva y de dominación el efecto de las desigualdades de poder entre los miembros de la pareja por diferencias en el status socioeconómico, medido por nivel de educación, ingresos y ocupación de las mujeres con respecto a los hombres ${ }^{13,14}$. Se concluye que, en el entorno en el que se producen los hechos violentos, la desigualdad entre los miembros de la pareja en términos de estatus en el empleo (por dedicación a tiempo completo o no y por tener un trabajo de primer orden -puestos ejecutivos, de dirección y administración- o no de las mujeres con respecto a los hombres) y en el ingreso también puede actuar como determinante en el problema.

\subsection{Desigualdad de género en el macrosistema}

La investigación sobre las actitudes, creencias y normas que impregnan la cultura en la violencia de género constituye una línea de trabajo fundamental para el diseño de estrategias eficaces de prevención primaria del problema. Un estudio reciente pone de manifiesto que el nivel de aceptabilidad de la violencia de género entre los países de la Unión Europea de 1999 es diferente, encontrando que todavía existen actitudes de culpabilización de las mujeres afectadas y de disculpa a los maltratadores ${ }^{15}$.

Dada la importancia de los factores del macrosistema para el diseño de intervenciones que permitan abordar el problema antes de que ocurra, se requiere de la realización de más estudios observacionales que puedan concretar

12. Hoffman, KL; Demo, DH; Edwards, JN. «Physical Wife Abuse in a Non Western Society: An Integrated Theoretical Approach». Journal of Marriage \& the Family 56 (1994), pp. 131-146.

13. Gage, AJ; Hutchinson, PL. «Power, Control and Intimate Partner Sexual Violence in Haiti». Archives of Sexual Behaviour 35 (2006), pp. 11-24.

14. Vieraitis, L; Williams, M. «Assessing the impact of Gender Inequality on Female Homicide Victimization Across U.S. Cities: A Racially Disaggregated Analysis». Violence Against Women 8 (2002); pp. 35-63.

15. Gracia, E; Herrero, J. «Acceptability of domestic violence against women in the European Union: a multilevel analysis». Journal of Epidemiology and Community Health 60 (2008), pp. 123-129.

Feminismo/s 18, diciembre 2011, pp. 291-299 
los mecanismos por los que las actitudes, creencias y normas pueden incrementar o reducir la probabilidad de malos tratos contra las mujeres.

\section{Conclusión}

La violencia de género contra las mujeres es un fenómeno complejo que se fundamenta en la interacción entre factores personales, situacionales y socioculturales. A pesar de la evidente dificultad que supone conciliar todas las aportaciones existentes, describir las causas de la violencia de género es imprescindible para mejorar la eficacia y la efectividad de las intervenciones en torno a este problema. El artículo de Lory L. Heise de 1998 constituye una primera aproximación a las causas de este problema, que resulta un gran estímulo para el desarrollo de hipótesis de investigación. El reto de los investigadores que lleven a cabo estos estudios en el futuro es abordar este amplio abanico de factores de la violencia de género aplicando diseños metodológicos que traten de mejorar la calidad de los estudios previamente realizados en torno a esta propuesta.

\section{Referencias bibliográficas}

ELLSBERG, Mary. «Violence against women and the Millennium Development Goals: Facilitating Women's access to support». Internacional Journal of Gynecology and Obstretics 94 (2006), pp. 325-332.

Gage, AJ; HutChinSON, PL. «Power, Control and Intimate Partner Sexual Violence in Haiti». Archives of Sexual Behaviour 35 (2006), pp. 11-24.

GRACIA, E; HERRERO, J. «Acceptability of domestic violence against women in the European Union: a multilevel analysis». Journal of Epidemiology and Community Health 60 (2008), pp. 123-129.

Gil-GonZÁlez, Diana; Vives-Cases, Carmen; Álvarez-Dardet, Carlos; LatourPÉREZ, Jaime. "Alcohol and intímate partner violence: do we have enough information to act?» European Journal of Public Health 16 (2006), pp 278-284.

Gil-GonZález, Diana; Vives-Cases, Carmen; Ruiz-Cantero, Maria Teresa; CARRASCO-PortiÑo, Mercedes; Álvarez-DARDET, Carlos. «Childhood experiences of violence in prepetrators as a risk factor of intimate partner violence: a systematic review». Journal of Public Health 30 (2007), pp. 14-22.

HEISE, Lory L. «Violence against women: An integrated, ecological framework» Violence against Women 4 (1998), pp. 262-290.

HOFFMAn, KL; Demo, DH; EDWARDS, JN. «Physical Wife Abuse in a Non Western Society: An Integrated Theoretical Approach». Journal of Marriage \& the Family 56 (1994), pp. 131-146. 
HOTALinG, GT; SUGARMAn DB. «An analysis of risk markers in husband to wife violence: the current state of knowledge». Violence and Victims 1 (1986), pp. 101-124.

JASINSKI, Jane: «Theoretical Explanations for Violence Against Women», en Renzetti, C; Edleson, J; Berger R (dirs.): Sourcebook on Violence against women, California, Sage Publications, 2001, pp. 5-21.

KrANTZ, Gunilla; GARCIA-MORENO, Claudia. «Violence against women». Journal of Epidemiology and Community Health 59 (2005), pp. 818-821

Ruiz-Cantero, Maria Teresa; Vives-Cases, Carmen; Artazcoz, Lucía; Delgado, Ana; García-Calvente, Maria del Mar; Miqueo, Carmen; Montero, Isabel; OrTIZ, Rocío; RondA, Elena; RuIZ, Isabel; VALLS, Carmen. «A framework to analyse gender bias in epidemiological research». Journal of Epidemiologic and Community Health 61 (Suppl 2), pp. 46-53.

Vieraitis, L; WiLliams, M. «Assessing the impact of Gender Inequality on Female Homicide Victimization Across U.S. Cities: A Racially Disaggregated Analysis». Violence Against Women 8 (2002); pp. 35-63.

WALBY, Sylbia. Theorising Patriarchy. Oxford, Blackwell, 1990. 